\title{
Clinical experience amongst surgeons in the Asymptomatic Carotid Surgery Trial-1 (ACST-1)
}

Short Title: Clinical experience in the Asymptomatic Carotid Surgery Trial-1 (ACST-1)

\section{Authors:}

Anne Huibers ${ }^{1,2}$, Djurre de Waard ${ }^{1,2}$, Richard Bulbulia ${ }^{3}$, Gert-Jan de Borst $^{2}$, Alison Halliday ${ }^{1}$, on behalf of the ACST collaborative group

\section{Affiliations:}

${ }^{1}$ Nuffield Department of Surgical Sciences, University of Oxford, Level 6 John Radcliffe Hospital, Oxford, OX3 9DU, United Kingdom

${ }^{2}$ Department of Vascular Surgery, University Medical Centre Utrecht, PO Box 85500, Utrecht, the Netherlands

${ }^{3}$ Clinical Trial Service Unit, Nuffield Department of Population Health, University of Oxford, Richard Doll Building, Old Road Campus, Roosevelt Drive, Oxford, OX3 7LF, United Kingdom

\section{Key words:}

Carotid Artery Stenosis; Carotid endarterectomy; clinical trials

\section{Word Count:}

2465 (including abstract and manuscript)

\section{Correspondence:}

Alison Halliday, Nuffield Department of Surgical Sciences, University of Oxford, Level 6 John Radcliffe Hospital, Oxford OX3 9DU, United Kingdom.

+44 (0)1865 221345 (phone)

+44(0)1865 221027 (fax)

alison.halliday@nds.ox.ac.uk 


\section{ABSTRACT}

\section{Introduction}

Hospital volume may influence the outcomes of carotid revascularization, but in trials the effect of the clinical experience of individual surgeons on procedural outcome is less certain. We assessed perioperative events rates amongst centres with different trial entry volumes and also the effects of individual operator experience in the first Asymptomatic Carotid Surgery Trial (ACST-1).

\section{Methods}

In 126 centres participating in ACST-1, surgeons were classified according to their in-trial experience (group A= 50 cases; group $B=51-100$ cases; group $C>100$ cases), centre enrolment volume (group $\mathrm{I}<30$ patients; group II 30-75 patients; group III>75 patients) and centre annual hospital volume (group 1<40 CEAs; group 2= 40-75 CEAs; group 3> 75 cases). Differences in perioperative event rates were compared using logistic regression analysis.

\section{Results}

In centres with most clinical experience compared with those with least experience (group C versus group A), the number of strokes or deaths was 8/275 (2.9\%) versus 24/810 (3.0\%); OR $0.99(95 \% \mathrm{Cl} 0.44-2.25, p=0.986)$. Numbers of strokes or death in high enrolment centres compared with low enrolment centres (Group III vs Group I) was $20 / 680(2.9 \%)$ versus $21 / 580(3.6 \%)$, OR $0.81(95 \% \mathrm{Cl} 0.43-1.51, p=0.921)$. In centres with a high annual volume compared with a low annual volume (group 3 versus 1), numbers of strokes and death was nonsignificantly lower 26/823 (3.2\%) versus 19/422 (4.5\%), OR 0.68 (95\% Cl 0.37-1.26, p=0.386). Cumulative stroke risk at 5- and 10-years were similar among different levels of reported clinical experience, enrolment volume and annual hospital volume.

\section{Conclusion}

Although our data did not demonstrate an association between perioperative complications and operators' experience, enrolment volume or annual hospital volume, rates of stroke or death were numerically lower in both 
high enrolment- and high annual volume centres. This lack of association could be explained by an overall low procedural risk in ACST-1. 


\section{INTRODUCTION}

Carotid endarterectomy (CEA) has been shown to reduce risk of subsequent stroke in patients with asymptomatic carotid disease.[1,2] The first Asymptomatic Carotid Surgery Trial (ACST-1) randomised over 3000 surgically suitable patients with a significant carotid stenosis to either immediate CEA plus appropriate medical therapy or to medical therapy alone. Allocation to immediate CEA halved the risk of future stroke (an absolute stroke risk reduction of around $6 \%$ in patients younger than 75 years of age).[1] This net benefit is partly determined by perioperative hazards, and whilst the peri-operative risk observed in ACST-1 was 3\%, this was offset by a lower longterm risk of stroke seen in participants allocated to immediate CEA. Better medical therapy (chiefly more widespread use of statins and at higher doses) might reduce long-term risk of stroke, but individual surgeon and institutional volume may also influence the peri-operative hazards of surgery.[3,4] Further data are necessary to determine the benefits of improved medical treatments, but large prospective registries and ongoing RCTs may provide data on complication rates amongst centres with variable experience and enrolment volume. Previous reports from RCTs often limit their analysis to centre enrolment volume, and the influence of individual experience of surgeons on peri-operative complications is unclear.[5,6] A reliable demonstration of a positive "volumeoutcome" relationship, especially from large registries, might suggest the need for a minimal annual caseload of CEA procedures per surgeon and/or centre. This would be of relevance to patients, referring physicians and agencies responsible for commissioning vascular services. In this study we assessed both the perioperative events rates and long-term stroke risk amongst centres with different volumes of enrolment and operators experience participating in the large ACST-1 trial. 


\section{METHODS}

\section{Patient selection}

The full trial protocol for ACST-1 has been described previously [ISRCTN 26156392].[7] Briefly, patients with significant "asymptomatic" carotid artery narrowing suitable for surgery were allocated to either immediate CEA or to deferral of any carotid procedure until it seemed to be more clearly needed (eg when they developed symptoms). Patients were suitable for randomization if: (1) unilateral or bilateral carotid artery diameter reduction $\geq 60 \%$ was present; (2) no neurological symptoms attributable to the carotid artery in the past 6 months had occurred; (3) the clinical condition of the patient allowed long-term follow up and (4) there was substantial uncertainty whether to choose immediate CEA plus medical therapy or medical therapy alone.

\section{Carotid endarterectomy and operators' requirements}

In patients allocated to immediate intervention, surgery was performed as soon as possible and the median duration from randomization to CEA was 1 month (interquartile range $0.3-2.5$ months). The choice of CEA technique (eg, general anaesthesia versus local anaesthesia; eversion CEA versus conventional CEA; shunting versus no shunting) was at the surgeon's discretion. In both treatment arms, patients received appropriate medical treatment, including anti-thrombotic (89\%), anti-hypertensive (70\%) and lipid-lowering therapy (45\%).

\section{Clinical experience}

In order to become a collaborator in ACST-1, each surgeon submitted a track record of symptomatic and asymptomatic patient outcomes, with a minimum caseload of 50 CEAs, and trial admission criteria of a 30-day stroke/death rate of $<6 \%$ (symptomatic patients) and $<4 \%$ (asymptomatic patients). During the trial, surgeons who had an unacceptably high stroke or death rate (as judged by the audit committee) were asked to stop recruiting, but no surgeon exceeded these limits. To monitor hospital volume outside the trial, a yearly questionnaire was sent to each centre asking for total numbers of CEAs performed in the preceding year. From these records perioperative event rates were compared for different levels of the following: 'reported clinical experience'; 'centre enrolment volume'; and 'annual hospital volume'. 'Reported clinical experience' was categorized into two groups. Group A consisted of hospitals in which the surgeons had declared their experience to be equal to the pre-defined 
required caseload of $50(=50)$. In Group B average experience per surgeon was 51-100 cases and in Group C average experience exceeded 100 cases. Centres were further classified according to numbers of patients enrolled in the trial: low (group I [<30 patients]); intermediate (group II [30-75 patients]); and high (group III [> 75 patients]). 'Annual hospital volume' was defined as the average number of CEAs each centre performed yearly during the trial period. Centres were considered as: low volume (group 1 [ 40 CEAs per year]); intermediate (group 2 [40-75 CEAs per year]); and high (group 3 [>75 CEAs per year]).

\section{Outcome events}

Before discharge, patients were assessed post-operatively by an independent stroke neurologist. They were followed up 4 and 12 months post randomization, and yearly thereafter. Outcome events (strokes, perioperative myocardial infarcts and any death) were reported to the central trial office. The Review committee adjudicated all trial events blinded to treatment allocation and surgeon identity, and any disagreement was resolved through discussion. The primary outcome event was peri-operative stroke or death (stroke or death that occurred within 30 days of surgery). Secondary outcome events were peri-operative MI, the composite endpoint of any perioperative stroke, death or $\mathrm{Ml}$ and 5- and 10 year overall stroke risk.

\section{Statistical analysis.}

Baseline characteristics are summarized with descriptive summary statistics. Differences in peri-operative event rates were compared using logistic regression analysis. Multivariate analysis was performed with variables indicating a p-value of $<0.30$ in univariate analysis. Kaplan Meier survival statistics were used to calculate cumulative rates of 5 and 10 year any stroke risk. Patients were studied from time of CEA and censored in case of stroke, death or if lost to follow-up. 


\section{RESULTS}

Amongst 3120 randomized trial patients, 1832 underwent a total of 1979 CEAs. In those allocated immediate surgery, 1532 CEAs were performed. A further 447 CEAs were performed in the deferred surgery arm for a variety of reasons (stroke or TIA, increase in carotid stenosis, patient or doctor changed their mind, prior to cardiac surgery or for non carotid-related symptoms).

\section{Prior clinical experience}

A total of 298 surgeons amongst 126 centres had track records approved. The mean CEA experience declared was 68 cases (range, 50-543). In 65 of 126 centres (52\%) the average experience of surgeons was equal to our minimal requested experience $(=50$ cases, group A). In the remaining 61 centres $(48 \%)$ average experience was higher than the minimum required (>50 cases, group $B$ ).

\section{Trial enrolment volume by centre}

Centre trial enrolment volume was as follows: 95/126 centres (75\%) randomized less than 30 patients (group I); $21 / 126$ centres (17\%) included 30-75 patients (group II); and 10/126 centres (8\%) included more than 75 patients (group III). The mean number of patients enrolled per centre was 25 (range, 1 - 194). Half of the centres (64/126, $51 \%)$ had one trial-approved surgeon, $36(29 \%)$ had two and $26(20 \%)$ had 3 or more. Table 1 shows baseline characteristics of patients by low, intermediate and high centre enrolment volume.

\section{Annual hospital volume during the trial period}

Average annual CEA volume per centre was 82 (range 6 - 460). Among participating centres 39/126 (31\%) performed less than 40 CEAs annually (group 1), 44/126 (35\%) between 40 and 75 CEAs annually (group 2). The remaining 43 (34\%) centres performed more than 75 procedures annually (group 3). The number of trial patients entitled in these groups were $n=422, n=587$ and $n=823$, respectively. In 47 out of 126 centres (37\%) low, medium or high 'in trial enrolment' corresponded with low, medium or high 'annual hospital volume'. In more centres (70/126 [56\%]) level of 'annual hospital volume' was higher than level of 'in trial volume'. 


\section{Procedural ( $<30$ days) outcomes}

The overall risk of peri-operative stroke or death was 3.3\%, comprising the following events: non-disabling stroke 26/1832 patients (1.4\%); disabling stroke 14/1832 (0.7\%); stroke death 13/1832 (0.7\%) nonfatal Ml 11/1832 (0.6\%); fatal MI 5/1832 (0.3\%); and other death 2/1832 (0.2\%). Secondary endpoints included any MI $(0.9 \%)$ and any $\mathrm{MI}$, stroke or death (3.8\%) (table $2 \mathrm{a}$ and figure 1 ). Odds ratios for these endpoints across different levels of reported clinical experience, enrolment volume and annual hospital volume are presented in table $2 \mathrm{~b}$.

\section{Long term outcome events}

The median duration of follow up for the 1832 patients studied was 70 months (interquartile range [IQR] 40 - 106 months). Table 3 shows the 5- and 10-year cumulative stroke risks among different levels of reported clinical experience, enrolment volume and annual hospital volume. Although not statistically significant, 10 year stroke rate was higher in low volume surgeons $(13.7 \%$ vs. $9.0 \%, p=0.242)$ and high enrolment centres $(14.6 \%$ vs. $8.3 \%$, $p=0.116)$ Cumulative overall survival at 10 years was higher in patients treated in medium (40-75 CEAs) volume centres $(57.7 \% ; 95 \% \mathrm{Cl} 50.1 \%-60.3 \%)$ than high (>75 CEAs) volume centres $(p=0.037)$. 


\section{DISCUSSION}

The present study describes peri-operative event rates amongst ACST-1 centres with different levels of enrolment volume and prior operator experience. In ACST-1, operative hazard was low, and we found no association between peri-operative complications, long term stroke risks and operators' experience, enrolment volume or annual hospital volume. Cumulative overall survival at 10 years was lower in patients treated in high volume centres.

Hospital volume, surgical experience, surgical technique and intraoperative monitoring have all been studied to determine if there was any association with complication rates following CEA.[4,8,9,10,11]We found risks of periprocedural complications to be lower in intermediate and high volume centres, but differences were not statistically significant. Thirty day mortality rates in NASCET and ACAS were low $(0.6 \%$ and $0.1 \%[2,12])$ and comparisons between "trial" hospitals (hospitals recruiting for both trials) and non-trial hospitals elsewhere in the United States, showed that trial results were not generalizable. Higher volume hospitals may have more vascular surgeons with a particular interest in carotid surgery as well as specialist anaesthetists and better short-term results.[13] Currently, European and US guidelines do not specify a minimum volume or experience for CEA practice.[14,15]

The present report does not demonstrate significantly lower complication rates amongst centres with more experienced surgeons. The impact of surgical experience on CEA outcome might be as important as hospital volume. In a recent systematic review, a subgroup analysis of operator experience showed that surgeons who operated infrequently performed better in high volume centres.[4] Others showed that individual surgeon experience is more important than hospital volume,[16] suggesting that patients would benefit more from referral to a high volume surgeon than to a high volume hospital. Our Audit Committee monitored surgeons' morbidity and mortality during the trial, and no surgeons had unacceptably high complication rates, possibly because there was a low overall procedural risk in ACST-1., making it less likely to show differences among the different levels of experience, enrolment and annual volume.

Amongst other trials studying enrolment volumes, in Carotid Revascularization Endarterectomy versus Stenting Trial (CREST), complication rates were not associated with centre enrolment volume.[6] In the StentProtected Angioplasty of the Carotid versus Endarterectomy (SPACE) trial enrolment volume was inversely related to peri-operative risks for Carotid stenting (CAS), but not for CEA.[5] The Endarterectomy versus Stenting 
in Patients with Symptomatic Severe Carotid Stenosis (EVA-3S) trial found that CAS risk was lower when performed by operators who did more than6 procedures per year, but the CEA hazard was so low that. However, in EVA-3S the absolute number of peri-procedural strokes or death after CEA was low (10 out of 262 cases) and data on hospital volume and risk was not reported.[17]

It is possible that surgeons may "pick" patients for randomized controlled trials and registries to avoid being classified as 'outliers'. The introduction of quality improvement can also lead to changes in patient selection (possibly excluding higher-risk patients, who may then be denied interventions for stroke prevention). The lower survival rate amongst high volume centres might be explained by performance bias. As high risk patients are more likely to be treated in high-volume centres. However, all cause mortality may be a relatively insensitive outcome measure. Therefore these findings should be interpreted with caution.

The present report has several limitations: Firstly, peri-operative hazards observed in ACST-1 were low, and these analyses may lack statistical power to detect an association between volume and outcome; second, surgeons collaborating in ACST-1 were relatively experienced, which may bias generalizability of our results; and finally, the experience reported in this study may not necessarily represent the life-time experience of a surgeon.

Reliable assessment of any volume - outcome relationship for peri-procedural hazards ideally requires analysis of large registries with tens of thousands of procedures and hundreds of relevant events.

\section{CONCLUSION}

No significant association was shown between peri-operative complications and enrolment volume or operators' experience in ACST-1. This may reflect the low number of events observed in the trial and selection bias, due to the credentialing procedures for surgeon selection.

\section{ACKNOWLEDGEMENTS}

We thank the patients who agreed to participate in the trial, and the many collaborating doctors and trial staff who worked hard to achieve reliable long-term follow-up. 


\section{SOURCES OF FUNDING}

The ACST-1 trial was funded by the UK Medical Research Council, the UK Stroke Association, and British United Provident Association Foundation. Professor Halliday's research is funded by the National Institute for Health Research (NIHR) Oxford Biomedical Research Centre based at Oxford University Hospitals NHS Trust and University of Oxford.

\section{DISCLOSURES}

The authors have no conflicts of interest. 


\section{REFERENCES}

1 Halliday A, Harrison M, Hayter E, et al. 10-year stroke prevention after successful carotid endarterectomy for asymptomatic stenosis (ACST-1): a multicentre randomised trial. Lancet 2010; 376:1074-1084.

2 Executive Committee for the Asymptomatic Carotid Atherosclerosis Study. Endarterectomy for Asymptomatic Carotid Artery Stenosis. JAMA. 1995; 273:1421-1428.

Archie JP Jr. Learning curve for carotid endarterectomy. South Med J 1988; 81:707-710.

Holt PJE, Poloniecki JD, Loftus IM, et al. Meta-analysis and systematic review of the relationship between hospital volume and outcome following carotid endarterectomy. Eur J Vasc Endovasc Surg 2007;33: 645651.

5 Fiehler J, Jansen O, Berger J, et al. Differences in complication rates among the centres in the SPACE study. Neuroradiology 2008; 50:1049-1053.

Gonzales NR, Demaerschalk BM, Voeks JH, et al. Complication rates and center enrollment volume in the carotid revascularization endarterectomy versus stenting trial. Stroke. 2014;45:3320-3324.

7 Halliday A, Thomas D, Mansfield A. The Asymptomatic Carotid Surgery Trial (ACST): Rationale and Design. Eur J Vasc Endovasc Surg 1994;8:703-710.

8 Caldwell K, Koch S, Khan I, et al. Impact of Surgical Specialty and Operator Experience on Outcomes Following Carotid Endarterectomy. J Vasc Surg 2015;61:577-578.

Rerkasem K, Rothwell PM. Patch angioplasty versus primary closure for carotid endarterectomy. Cochrane Database Syst Rev 2009;4.

10 Rerkasem K, Rothwell PM. Routine or selective carotid artery shunting for carotid endarterectomy (and different methods of monitoring in selective shunting). Cochrane Database Syst Rev 2014;23.

11 De Borst GJ, Moll FL. Evidence overview for shunting, patching, type of endarterectomy and anesthesia during carotid surgery. J Cardiovasc Surg (Torino) 2014;55:1-9.

Eliasziw M, Rankin RN, Fox AJ, et al. On behalf of North American Symptomatic Carotid Endarterectomy Trial (NASCET) group. Accuracy and Prognostic Consequences of Ultrasonography in Identifying Severe Carotid Artery Stenosis. Stroke 1995;26:1747-1752.

13 Wennberg DE, Lucas FL, Birkmeyer JD, et al. Variation in carotid endarterectomy mortality in the Medicare population: trial hospitals, volume, and patient characteristics. JAMA 1998;279:1278-1281.

14 Kakisis JD, Avgerinos ED, Antonopoulos CN, et al. The European Society for Vascular Surgery guidelines for carotid intervention: an updated independent assessment and literature review. Eur J Vasc Endovasc Surg 2012;44:238-243. 
vertebral artery disease: executive summary. A report of the American College of Cardiology Foundation/American Heart. Circulation 2011;124:489-532.

16 Cowan JA Jr, Dimick JB, Thompson BG, et al. Surgeon Volume as an Indicator of Outcomes after Carotid Endarterectomy: An Effect Independent of Specialty Practice and Hospital Volume. J AM Coll Surg 2002;7515:814-821.

17 Mas JL, Chatellier G, Beyssen B, et al. Endarterectomy versus Stenting in Patients with Symptomatic Severe Carotid Stenosis. N Engl J Med 2006;355:1660-1671. 